\title{
Note préliminaire concernant la transmission de Stilesia Globipunctata (RIVOLTA, 1874) du mouton par divers acariens oribates *
}

\author{
por M. GRABER ef J. GRUVEL \\ (Avec la coll. technique de $M^{\mathrm{me}} \mathrm{J}$. LANUSSE)
}

\begin{abstract}
RÉSUMÉ
En mars-avrıl 1964, les auteurs ont réussı à transmettre Stilesia globipunctoła à des moutons préalablement déparasités, à partir d'Oribates recueillıs en trois points differents de la concession du Laboratoire de Farcha (Tchad). L'une des zones avait été préalablement infestée au moyen d'œufs de Cestodes largement répandus sur le sol.

Sur les 39 moutons mis en expérience, 17 ont réagi positivement.

Les Oribates, hôtes intermédiaires appartiennent d'une part à la farmilie des Scheioribatidae et aux espèces Scheloribates perforatus (WALLWORK, 1964) et Scheloribates parvus conglobatus (WALLWORK, 1964), d'autre part à la famille des Ceratozetidae, et, selon toute vraisemblance, à l'espèce Africacarus colcaratus (WALLWORK, 1964).

Les Cysticercoïdes découverts qu cours de la dissection des Scheloribates perforalus parasités se présentent sous l'aspect d'une masse ova ide ou sphérique, entourée d'une double enveloppe exiérieure plus ou moins bien délimitée. Ils mesurent de $96-97 \mu \times 90$ à $180-200 \mu \times 140 \mu$. L'invagination céphalique est peu distincte et les ventouses sont assez visıbles à un fort grossissement.

Le taux d'infestation des Acariens est faible : de 7 à 12,5 p. 1000 dans ies conditions naturelles et 39,5 p. 1000 dans les conditıons expérimentales. La mise en évidence el l'énucléation des Cysticerco ides noyés dans les tissus de l'hôte s'avère particulièrement laborieuse.
\end{abstract}

\section{INTRODUCTION}

Depuss la découverte du cycle évolutif de Moniezia expanso par STUNKARD (1937), de nombreux auteurs se sont penchés sur le problème des Acariens Oribates, hôtes Intermédiaires des divers Anoplocephalidae qui sont à l'origine du Téniasis des ruminants domestiques et sauvages.

La plupart des recherches concernent les Oribates vecteurs de Moniezio expansa et de

(*) Nous tencns d̀ remercier vivement M. J. A. WALLWORK, Dépariment of zoology, Westfield college, London, d'avoir bien voulu assurer la délermination des acariens oribates recueillıs.
Moniezio benedenl. Elles ont été réalisées aux U.S. A. (STUNKARD, 1937 et 1944 ; STOLL, 1938 ; KRULL, 1939 ; KATES et RUNKEL, 1947 ef 1948 ; EDNEY ef KELLEY, 1953 ; WALLWORK et RODRIGUEZ, 1961), en U. R. S. S. (POTEMKINA, 1944 et 1959 ; SHALDININA, 1953 ; PETROCHENKO, 1954 ; ANTIPIN et ERSHOV, 1956 ; BOEV ef ORLOV, 1958 ; OREKHOV, 1960 ; SOKOLOVA et PANIN, 1960 ; SVADSHYAN, 1962), en Angleterre (RAYSKI, 1949), (en Australie (ROBERTS, 1953), en France (MOREL, 1953), au Canada (RAO et CHOQUET- 
TE, 1951), en Yougoslavie (RUKAVINA, 1960), en Pologne (RAJSKI, 1959), aux Indes (MEHRA ef SRIVASTATA, $1955 a$ et $b$ ) et au Japon (WATANABE, 1957).

Actuellement, il existe plus de 40 espèces d'Oribates capables de transmettre le Ténıasis à $M$. expansa ef à $M$. benedeni. Quant aux hôtes intermédiaires des Thysanosominae, ils sont encore très mal connus. Les quelques essais effectués intéressent le cycle évolutif de Thysanosoma actinioides (ALLEN, 1959), de Thysaniezia ovilla et d'Avitellina centripunctoto (POTEMKINA, 1944 ; NADAKAL, 1950 ; SOKOLOVA ef PANIN, 1960 ; KUZNETZOV, 1962).

Le but du présent traval est de donner un certain nombre de renseignements préliminaires sur le cycle évolutif de Stilesia globipunctata (RIVOLTA, 1874), Cestode qui frappe 32 p. 100 des moutons de la République du Tchad (GRABER ef SERVICE, 1964).

\section{MATÉRIEL ET MÉTHODE}

\section{Les lieux de récolte}

Situés à l'intérieur de la concession du Laboratoire de Farcha, ils ont été choisis en fonction de leur richesse en Oribates. Ceux-ci ont été recueillis en trois points différents :

a) A partir d'un terrain lıbre (ZI) situé au bord du Chari et servant de déversoir à la stafion de relevage du Laboratoire. Le tapis végétal épais qui subsiste toute l'année est fort prisé des moutons dont beaucoup sont porteurs d'Anoplocopholidae, d'où possibilité d'infestation accrue des hôtes intermédiaires présents dans le sol.

b) A partir d'une surface ( $Z 2)$ fortement protégée par un gros Tamorindus indica. Très fréquentée par des moutons parasités au cours de 1962, elle a été clôturée en décembre de la même année, de manière d̀ éviter les incursions intempestives qui auraient pu gêner ultérieurement la bonne marche de l'expérience.

c) à partir d'une troisıème aire (Z 3) également clôturée et faiblement ombragée par un Kahaya senegalensis. Cette aire a reçu en décembre 1962 environ cinq millions d'œufs de Stilesio globipunctoto provenant du broyage de fragments mûrs de Cestodes. Les œufs, mélangés à l'humus, ont été inégalement dispersés à la surface du sol. Celui-ci a été arrosé toute la saison sèche suivante et couvert de débris végétaux (feuilles mortes) ce qui a permis la survie des Oribates particulièrement nombreux en cet endrolt.

\section{$2^{\circ}$ Les animaux d'expérience}

39 moutons originaires de Fort-Lamy ont été utilisés pour les infestations expérimentales. Vu l'incidence élevée du Téniasis ovın dans la région, les animaux ont subi un traitement préalable au moyen de Cestodifuges actifs sur Stilesio globipunctata : dans un premier temps its ont reçu entre 500 et $1.000 \mathrm{mg}$ d'Arséniate d'Etain par tête, et quelques jours plus tard, de 50 à 100 $\mathrm{mg} / \mathrm{kg}$ de 14.970 R. P. ou de 14.015 R. P. On sait que I'Arséniate d'Etain, à cette dose, suffit à assurer amplement la destruction de Stilesia globipunctota (CASTEL, GRABER, GRAS ef CHHAY-HANCHENG, 1960).

Le second traitement par les nouveaux ténifuges Rhône-Poulenc n'a été institué que pour obtenir une sécurité absolve. Les résultats se sont montrés favorables, puisque les neuf moutons témoins, soumis à ce double traitement, n'ont présenté, à l'autopsie, que des nodules vides, sans scolex, ni Cestodes.

Les animaux ainsi préparés, ont été placés dans des stalles cımentées et nourris, dès leur arrivée, avec du fourrage vert coupé sur les bords du Chari dans des zones sablonneuses désertes. La présence ou l'absence d'Oribates dans les herbes a été contrôlée rigoureusement tous les jours et pour chaque botte. Plus tard, des branches d'arbre, également contrôlées, ont été ajoutées à la ration.

\section{Techniques ef époques d'infestation}

La mise en évidence des Acariens se fait par lavage ou chauffage des mottes de terre prélevées dans l'une ou l'autre des trois zones dont il a été question plus haut.

L'infestation ne souffre pas de difficulté. Les Oribates, une fois comptés et déterminés, sont administrés au mouton dans de l'eau « à la bouteille $\gg$.

Les infestations expérimentales ont eu lieu en octobre-décembre 1963 pour Z2, de décembre 1963 à mars 1964 pour $Z 1$ et de janvier à juin 1964 pour Z3. 


\section{RÉSULTATS}

\section{A. - Zone $n^{\circ}$ III $\left(Z_{3}\right)$}

Les résultats figurent au tableau $n^{\circ}$ l.

\section{Discussion.}

a) Les Oribates décelés dans ce terrain appartiennent, dans leur grande majorité, à la famille des Scheloribotidae et à deux espèces différentes: Scheloribates perforatus (WALLWORK, 1964) ef Scheloribates parvus sp. conglobotus (WALLWORK, 1964).

If s'agit d'Acariens plus ou moins ovoides, de petite taille $(0,5-0,6 \mathrm{~mm})$ et de couleur foncée. Ils ont tendance à vivre groupés dans le sol, en formant des colonies quelquefois assez importantes.

Des fluctuations saisonnières semblent exister dans la répartition des deux espèces en cause : dans les conditions de l'expérience, S. perforatus est l'espèce dominante de janvier à avril. En mai, elle disparaît presque totalement pour faire place à 5 . parvus conglobatus qui peut alors être recueilli en grande abondance. Des sondages ultérieurs permettront de dire si cette hypothèse est valable ou non.

b) Le taux d'infestation de Scheloribates perforatus par les Cysticercoides de Stilesia globipunctato est assez faible : lors des infestations expérimentales, il ne dépasse 39,5 p. 1000 (250 scolex au total pour 6.321 Oribates administrés). La dissection d'exemplaires de la même espèce, prélevés dans la même zone, donne des résultats encore moins favorables ( 6 p. 1.000 des 1.380 Scheloribates examinés). Scheloribates parvus conglobatus serait plutôt un vecteur accidentel, comme semble le démontrer le médiocre pourcentage d'Acariens infestés ( 3 sur 1.465, soit 2 p. 1.000).

La faible quantité d'Oribates parasités s'explique vraisemblablement par l'absence de maturité d'un certain nombre d'œufs de Stilesia répandus sur $Z 3$, la destruction de quelqu'uns d'entre eux por les agents extérieurs (chaleur et sécheresse), la mortalité plus ou moins élevée des hôtes intermédiaires infestés et par l'aptitude d'Oribates de 0,5-0,6 mm à absorber des Oncosphères de Stilesıa de 50-60 $\mu$. Peut-être faut-il incrimıner la répartition irrégulière du matériel infestant à la surface de Z3: la plupart des
Acariens porteurs de Cysticercoides ont été trouvés là où la couche d'humus, renfermant les œufs de Stilesia, avait, au départ, été la plus épaisse.

II n'en demeure pas moins vrai que l'infestation expérimentale de certains Orıbates à portir des œufs de Thysonosominae paraît difficile. NADAKAL (1960) met en contact des Protoscheloribates et des Trichoribates avec des milliers d'oncosphères d'Avitellına centripunctata: il n'obtıent que 5 Cysticercoüdes dans le premier cas et rien dans le second.

La différence avec les Cestodes du groupe Moniezia est donc considérable. KATES et RUNKEL (1948), sur un terrain artificiellement infesté par des œufs de Moniezia expansa, observent des taux d'infestation allant jusqu'à $340 p$. 1.000 pour Galumno virginiensis, 110 p. 1.000 . pour Galumna emarginatus et 60 p. 1000 pour Oribatula minuta.

Jusqu'à plus ample informé, les œufs de Stilesio globipunctata ne sont capables de contaminer qu'un petit nombre de Scheloribotes perforatus, d̀ l'endroit même où sont tombées les déjections des moutons atjeints.

c) En mars 1964 - soit 15 mois après l'infestation artificielle du terrain - les Cysticercoïdes mûrs, découverts, après dissection de Scheloribates infestés, se présentent sous deux formes différentes :

- les uns mesurent $96-97 \mu \times 90 \mu$.

- les autres $180-200 \mu \times 140 \mu$.

D'aspect sphérique ou ovoide, ils sont caractérisés par une double enveloppe extérieure plus ou moins bien délimitée, mais toujours moins nette que l'enveloppe correspondante des Cysticercoïdes de Moniezıa, par une invagination céphalique peu distincte et par quatre ventouses assez visibles à un fort grossissement (Phot. I et II ; fig. 1 et 2).

Ils adhèrent profondément aux tissus de l'hôte et leur énucléation s'avère partıculièrement difficile, ce qui n'est pas le cas des formes larvaires de Moniezio.

\section{B. - Zones I $\left(Z_{1}\right)$ et II $\left(Z_{2}\right)$}

$10 Z 1$ (Tab. II)

2० Z 2 (Tab. III) 
TABLEAU No

\begin{tabular}{|c|c|c|c|}
\hline $\begin{array}{l}\text { Mout on } \\
n^{\circ}\end{array}$ & $\begin{array}{l}\text { Cestodes élimenés } \\
\text { après traltement }\end{array}$ & $\begin{array}{l}\text { Nombre d'Oribates } \\
\text { admunistrés }\end{array}$ & $\begin{array}{c}\text { Cestodes prósents à } \\
\text { I'autopsie }\end{array}$ \\
\hline 1 & Moniezla & $395+$ & 0 \\
\hline 2 & 0 & $375+$ & 0 \\
\hline 3 & Avitellina & $194+$ & 0 \\
\hline 4 & 0 & $200+$ & 0 \\
\hline 5 & Maniezia & $257+$ & $\begin{array}{l}25 \text { nodvies S. globipunctata at } \\
25 \text { scolex }\end{array}$ \\
\hline 6 & 0 & $277+$ & 0 \\
\hline 7 & 0 & $211+$ & 0 \\
\hline 8 & Avitellina & $236+$ & 0 \\
\hline 9 & 0 & $197+$ & 0 \\
\hline 10 & 0 & $422+$ & 0 \\
\hline 11 & 0 & $312+$ & 11 nodules et 11 scolex \\
\hline 12 & Moniezia & $402+$ & 0 \\
\hline 13 & 0 & $284+$ & 12 nodules et 12 scolex \\
\hline 14 & 0 & $372+$ & 94 nodules at 94 scolex \\
\hline 45 & 0 & $244+$ & 1 nodule et 1 scoler \\
\hline 16 & 0 & $507+$ & 80 nodules et 80 scoler \\
\hline 17 & 0 & $433+$ & 26 nodules et 26 scoler \\
\hline 18 & 0 & $523+$ & 0 \\
\hline 19 & 0 & $4 \mathrm{BO}+$ & 0 \\
\hline 20 & 0 & $518++$ & 3 nodule et 3 scolex \\
\hline 21 & 0 & $298++$ & 0 \\
\hline 22 & 0 & $314+t$ & 0 \\
\hline 23 & 0 & $335++$ & 0 \\
\hline
\end{tabular}

+ Scheloribates perforatus (Wellwork, 1964)

++ Soheloribates parvus sap. canglobatus (Wallwork, 1964)

TABLEAU NOII

\begin{tabular}{|c|c|c|c|}
\hline $\begin{array}{l}\text { Mouton } \\
n^{\circ}\end{array}$ & $\begin{array}{l}\text { Cestodes éliminés } \\
\text { après traitement }\end{array}$ & $\begin{array}{l}\text { Nombre d'Oribates } \\
\text { administrés }\end{array}$ & $\begin{array}{c}\text { Cestodes préserts à } \\
\text { l'eutopsia }\end{array}$ \\
\hline 1 & 0 & 187 & 0 \\
\hline 2 & 0 & 228 & 0 \\
\hline 3 & 0 & 302 & $\begin{array}{l}1 \text { godule S. globupunctata et } \\
1 \text { scolex }\end{array}$ \\
\hline 4 & 0 & 279 & $\begin{array}{l}4 \text { nodules S. globspunctata et } \\
4 \text { scolex }\end{array}$ \\
\hline 5 & Moniezia & 238 & 0 \\
\hline 6 & 0 & 266 & $\begin{array}{l}12 \text { nodules } 5 \text {. globlpunctata et } \\
12 \text { scolex. }\end{array}$ \\
\hline 7 & Avitellina & 56 & $\begin{array}{l}2 \text { noduzes } S \text {. globlyunctate et } \\
2 \text { scolex }\end{array}$ \\
\hline TOTAL & & 1.456 & \\
\hline
\end{tabular}


Retour au menu

TABLitA N N $^{\circ}$ III

\begin{tabular}{|c|c|c|c|}
\hline $\begin{array}{c}\text { Mouton } \\
n^{\circ}\end{array}$ & $\begin{array}{l}\text { Cestodes éliminés } \\
\text { après traitement }\end{array}$ & $\begin{array}{l}\text { Nombre d" Oribates } \\
\text { adminzatrés }\end{array}$ & $\begin{array}{l}\text { Ceatodes présents à } \\
\text { l'eutopsie }\end{array}$ \\
\hline 1 & Avitelline & 340 & 1 \\
\hline 2 & Aritellina & 300 & 0 \\
\hline 3 & 0 & 300 & $\begin{array}{l}5 \text { nodules s. globipunctata et } \\
5 \text { scoler }\end{array}$ \\
\hline 4 & Avitellina & 346 & $\begin{array}{l}6 \text { nodules } S \text {. Elobipunctate et } \\
6 \text { scoler }\end{array}$ \\
\hline 5 & Monlezia & 200 & 0 \\
\hline 6 & Avitellina & 300 & 0 \\
\hline 7 & Moniezia & 300 & $\begin{array}{l}1 \text { nodule S. Elobipuncteta et } \\
1 \text { scolex }\end{array}$ \\
\hline 8 & 0 & 497 & $\begin{array}{l}1 \text { nodule S. Elobipunctata et } \\
1 \text { scolex }\end{array}$ \\
\hline 9 & 0 & 396 & $\begin{array}{l}7 \text { nodules S. globpunctata et } \\
7 \text { scolex }\end{array}$ \\
\hline TOTAL & & 2.979 & \\
\hline
\end{tabular}

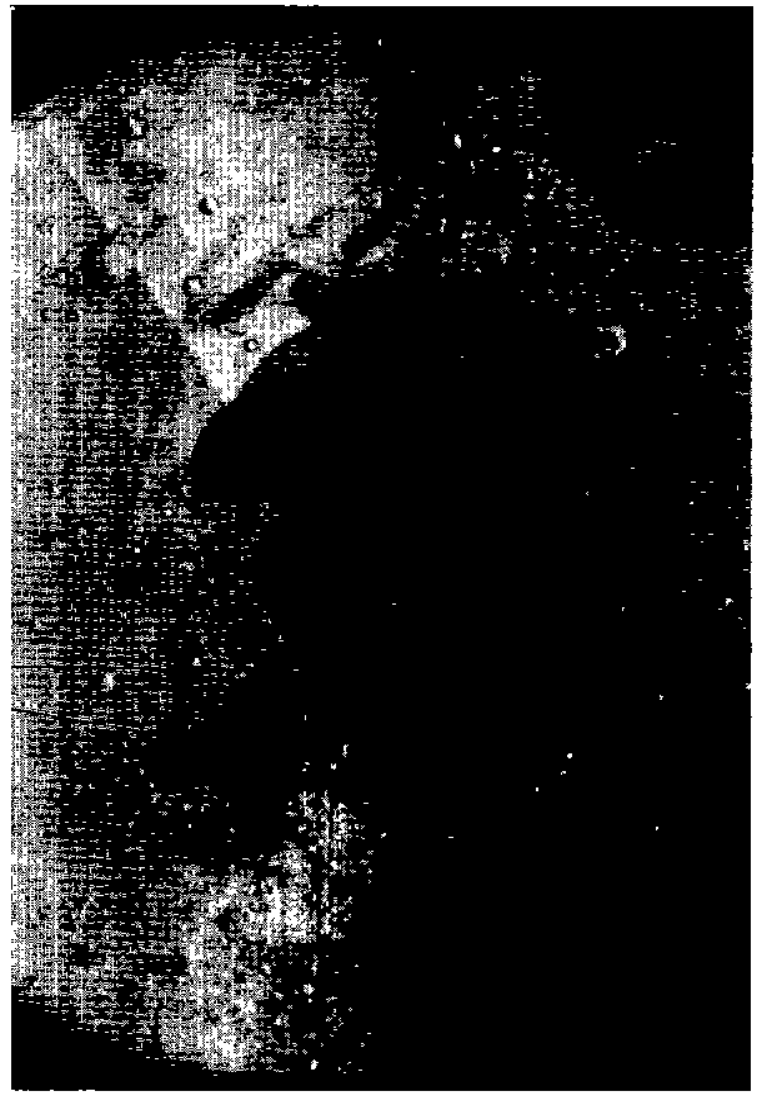

Scheloribales perforatus montrant deux cysticercoîdes énuclées $(X 120)$

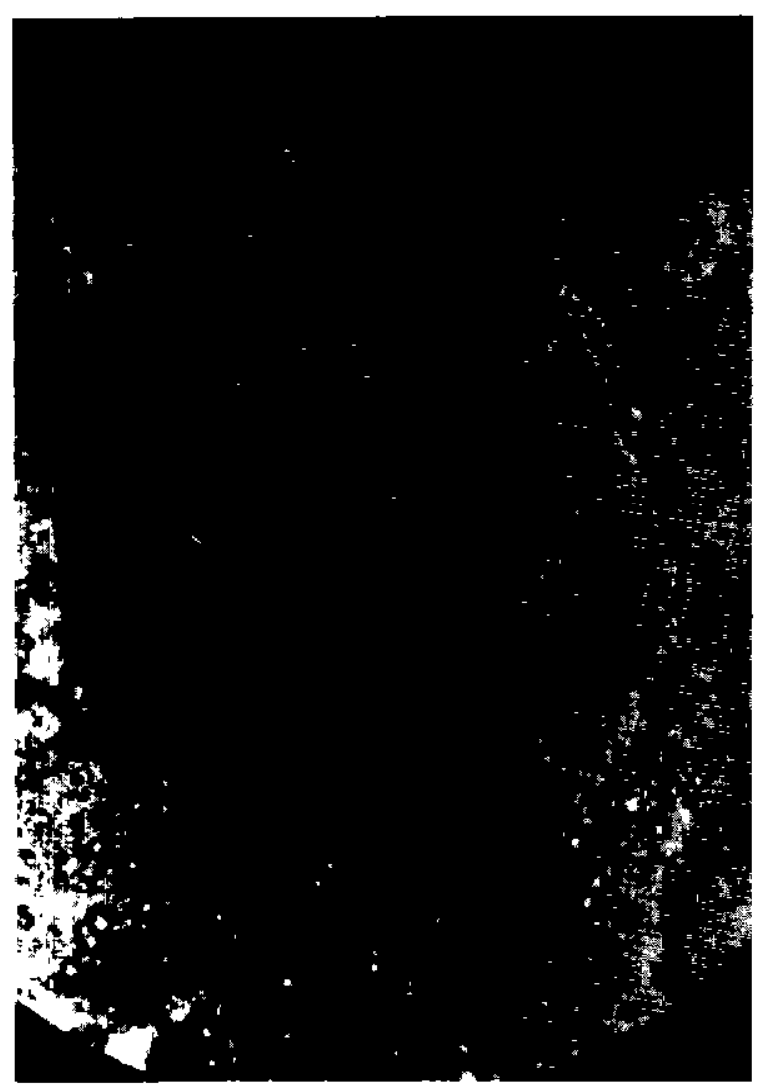

Cysticerccide de stilesia globipunctata. On devine les quatre ventouses ( $X 600$ ) 

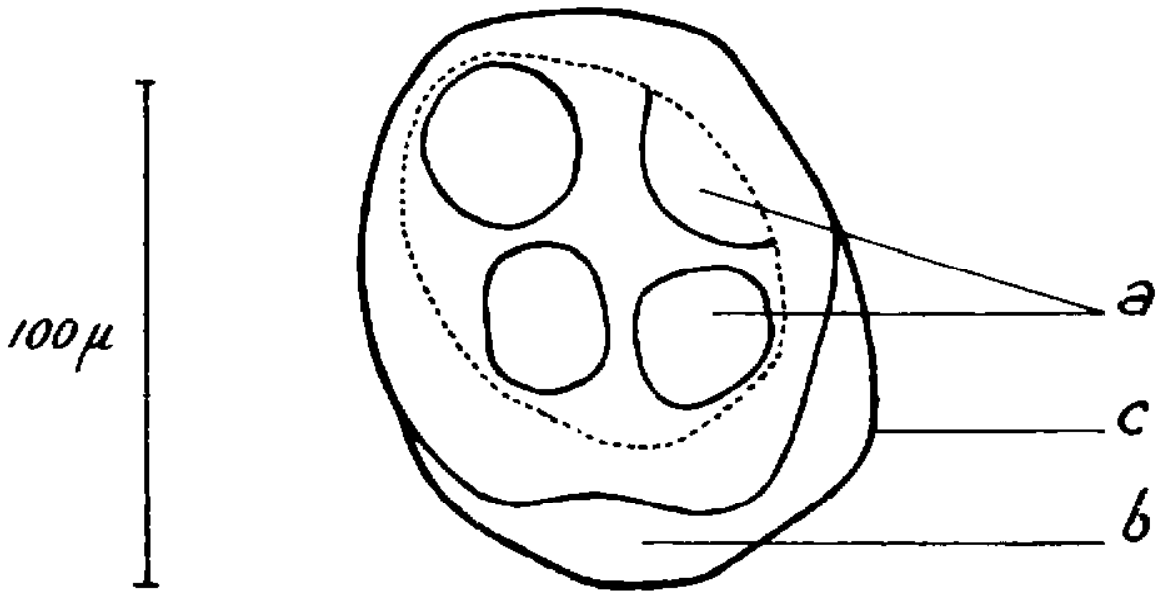

Fig. 1. - Cysticercoide de Stilesia globifunctata (à partir de Scheloribotes perforotus)

a) Ventouses, b) invagination céphalique, c) enveloppe extérieure.
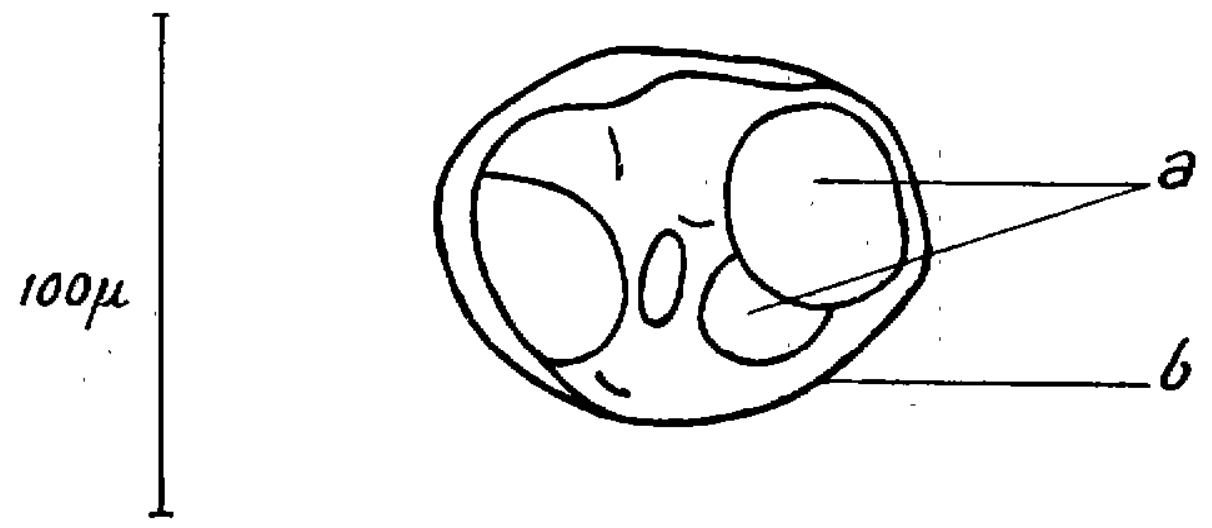

Fıg. II. - Cystıcercoıde de Stilesia globifunclata (ò partir de Schelaribotes perforatus) a) Ventouses, b) enveloppe extérieure . 


\section{DISCUSSION}

a) Les Acariens infestés des zones 1 et 2 paraissent devoir être rattachés à la famille des Ceratozetidae dont la détermination des espèces est en cours. Sous réserve de vérification ultérieure, I'une d'entre elles, Africacarus calcarotus (WALLWORK, 1964) pourrait être retenue comme vecteur possible.

Les Ceratozetidés sont des Acariens globuleux, brun foncé, portant parfois à la partie antérıeure du Notogaster une étroite zone circulaire plus claire coiffée latéralement de deux petıts points rouges. Ils sont beaucoup moins grégaires que les Scheloribates.

b) Le taux d'infestation des Ceratozetes de Z 1 $(12,5$ p. 1.000$)$ régulièrement en contact avec des œufs mûrs de Stilesio, est supérieur à celui de Z2 (7 p. 1.000). La zone 2, ayant été complètement isolée en décembre 1962, aucun mouton n'a pu y pénétrer après. Au moment de l'expérience, les Acariens porteurs de Cysticercoïdes l'étaient donc depuis plus d'un an, ce qui correspond aux observations de STOLL (1935), EUZÉBY (1957) ef POTEMKINA (1959) concernant la longévité des formes larvaires chez l'hôte intermédiaire.

c) L'infestation d'ovins réalisée à partır de Cerotozetıdae parasités, laıssés dans leur milieu naturel sans aucune modification $(Z 1)$, est assez régulière et - semble-t-ıl peu massive.

d) La transmıssion de Stilesia glabipunctata au mouton est fonction des fluctuations saisonnières subies par les diverses populations d'Oribates rencontrées : d'octobre à jonvier, elle est le fait des Ceratozetes ; de janvier à mai, elle dépend de Scheloribotes perforatus et en juin de Scheloribates porvus conglobotus.

Ces observations provisorres faites au Laboratoire paraissent donc indiquer que plusieurs espèces d'Oribates, hôtes intermédiaires certains, sont susceptibles de se relayer à tour de rôle dans le courant de l'année, de manière à assurer la pérennité et la constance de l'infestation par Stilesia globipunctata. L'épidémiologie de la Stilesio confirme d'ailleurs ce fait (GRABER et SERVICE, 1964) : Il n'y a pas de «trou» dans la répartition annuelle de ce Téniasis ef le nombre d'animaux parasités demeure sensiblement égal, quelle que soit la saison envisagée.

\section{CONCLUSION}

Jusqu'à ce jour, la recherche du Cycle évolutif des Anoplocephalidae, agents du Téniasis des rumınants, a surtout intéressé des espèces cosmopolıtes telles que Moniezio expanso et Moniezıa benedeni.

Par contre, les hôtes intermédiaires des Thysonosominae, si fréquents dans certaines parties du monde, sont encore très mal connus et les quelques études récemment parues ne concernent que Thysanosoma Actiniordes, Thysaniezia ovilla et Avitellina centripunctata.

Au cours de l'année 1963, les auteurs ont réussi, au moyen d'Oncosphères mûres de Stilesia globipunctata largement répandues sur le sol, à infester une petite surface riche en Oribates.

Au printemps 1964, ces Acariens, donnés à des moutons préaláblement traités à l'Arséniate d'Etain et maintenus en dehors de toute source de contage, ont entraîné l'apparition d'un certain nombre de Stilésia globipunctata chez 8 des 23 animaux mis en expérience.

Scheloribates perforatus et Scheloribates parvus conglobatus (WALLWORK, 1964) n sp. semblent être les espèces responsables de cette transmission.

A la dissection, ont été découverts des Cysticercoìdes complètement développés. D'aspect ovoide ou sphérıque et de taille variable ( 97 à $200 \mu$ ), its présentent - comme les formes larvaires de Moniezia expansa - une double enveloppe extérieure pas toujours très nette, une invagination céphalique peu distincte et quatre ventouses plus ou moins visibles.

La mise en évidence des Cystıcercoides dans le corps de l'Acarien est particulièrement difficile.

Le taux d'infestation de Scheloribotes perforatus est falble : environ 39,5 p. 1,000. D'autres essais de transmission au mouton, effectués à partir d'Oribates récoltés dans des sols soumis à une infestation naturelle antérieure, ont montré que d'autres hôtes intermédiaıres, appartenant vraisemblablement à la famille des Ceratozetidae, pouvaient, en matière de Stilesiose, remplir le même rôle que $S$. perforatus. Le pourcentage de Ceratozetes parasités varie de 7 à 12,5 p. 1.000 . 


\section{SUMMARY}

\section{A preliminary note concerning the transmission of Stilisio globipunctato} (Rivolta, 1874) of the sheep by various mites

In march-april 1964, the authors succeeded in transmitting Stilesia globipunctato to previously disparasited sheep, from Oribates collected at three different points of the Farcha (Lake Chad) Laboratory concession.

One of the zones had previously been infested by means of Cestodes eggs, widely spread out on the soil.

Out of the 39 tested sheep, 17 have reacted positively.

The Oribates, intermediary hosts, belong on the one hand to the Scheloribatidae family and to Scheloribates perforatus (WALLWORK, 1964) and Scheioribates porvus conglobatus (WALLWORK, 1964), and on the other hand, they belong most likely, to the Africacorus colcaratus species (WALLWORK. 1964).

The Cysticercoindes discovered during the dissection of parasited Scheloribotes perforatus appear as an ovoid, spherical mass, surrounded by a double outer enveloppe, more or less well delimited. They measure from $96-97 \mu \times 90$ to $180-200 \mu \times 140 \mu$. The cephalic invagination is not very clear and the cuppingglasses are visible enough at a strong enlargement.

The infestation rate of Acariens is low: from 7 to 12,5 por 1000 under natural conditions, and of 39,5 por 1000 under experimental conditions. The showing off and enucleation of the Cystercoides drowned in the host's tissues, appears to be particularly hard.

\section{RESUMEN}

Nota preliminar en cuanto a la transmisión de Stilesia globipunctata (Rivolta, 1874) de la oveja por diferentes acaridos oribatidos

En marzo-april de 1964, los autores lograron para transmitir Stilesia globipunctata a ovejas previamente deparasiladas, a partir de Oribatos recogidos en tres sitios diferentes del territorio de Farcha (Tchad). Una de las zonas habia sido previamente infectada mediante huevos de céstodos ampliamente distribuidos por el suelo.

En las 39 ovejas experimentadas, 17 reaccionaran positivamente.

Los Oribatos, huéspedes intermediarios pertenecen ya a la familia de los Scheioribatidas y a las dos especies Scheloribotes perforatus (WALLWORK, 1964) y Scheloribates parvus conglobotus (WALLWORK, 1964), ya à la familia de lo5 Ceratozetidos, $y$, verosimilmente, a la especie Africacarus calcoratus (WALLWORK, 1964).

Los cisticercoides descubiertos en disección de los Scheloribotes perforatus se presentan bajo forma de una masa ovoide o esférica, con una doble envoltura exterior más o menos limitado. Meden de $96-97 \mu \times 90 \mu$ a 180-200 $\mu \times 140 \mu$. La invaginación cefálica es poco distinta y los ventosos son bastante visibles cón un importante aumento.

El termino media de infección de los acáridos es poco importante : de 7 a 12,5 por 1000 en las condiciones naturales y de 39,5 por 1000 en las condiciones experimentales. La busqueda y la enucleación de los cisticercoidos en los tejidos del huésped es particularmente dificil. 


\section{BIBLIOGRAPHIE}

1. NAEGELE (1964). - Advances in Acarology. $480 \mathrm{p}$.

2. ALLEN (R. W.), (1959). - Preliminary note on the larval development of the fringed Tapeworm of sheep, Thysanosoma actinioides in Psocids. J. Parasit., 45, 5, 537-8.

3. ANANTARAMAN (M.) (1948), - Oribatid mites and their economic importance. Nature CLXI, 409-10.

4. ANANTARAMAN (M.) (1951). - Development, of Moniezia. Sci. Cult., 17,'155-7.

5. ANTIPIN (D. N.) et ERSHOV (V. S.) (1956). Parasitology and parasitic diseases of livestock. Moscous-Israel Prog. Scl. Trans., 1960.

6. BAKER et WHARTON (195 o). - An introduct to Acarology New York. $465 \mathrm{p}$.

7. BOEV (S. N.) et ORLOV (N. P.) (1958). Les maladies parasitaires des animaux d'élevage au Kazakstan et les moyens de les combattre. Bull. Off. Int. Epiz., XLIX, 11/12, 189-90.

8. CASTEL (P.), GRABER (M.), GRAS (G.) et CHHAY-HANCHENG (1960). - Action de l'Arséniate d'Etain sur divers Cestodes de mouton. Rev. Elev. Méd. Vét. Pays Trop., 13, 1, $57-74$.

9. EDNEY (J. M.) et KELLEY (G. W.) (1963). Some studies on Galumna virginiensis and Moniezia expansa. J. Tennessee Acad. Scie., 28, 4, 287-96.

10. EUZÉBY (J.) (1957). — Le Téniasis des ruminants et son traitement. Rev. Méd. Vét., 20, 3, 178-184.

11. FUKUI (M.) (1960). - Studies on Moniezia expansa and its intermediate host - IV : Survey of Moniezia at a sheep run in the suburds of Tokyo. J. Pap. Vet. Med. Ass., 13, 5, 214-8.

12. GRABER (M.) (1959). - Les Anoplocephalidae et les affections qu'ils provoquent chez les animaux domestiques. Coll. I. A. C. E. D./ C. C. T. A. Helm. Anim. Dom., no 49, 80-130.

13. GRABER (M.) et SERVICE (J.) (1964), - Le Téniasis des bovins el des ovins de la République du Tchad. Quelques données épidémiologiques intéressant les zones sahéliennes. Rev. Elev. Méd. Vét. Poys Trop. (en préparation).
14. HONESS (R. F.) (1954). - Studies on the life history of Thysanosoma actinioides. Conf. Parasit. Anim. Dom. Montano, U. S. A., 18-20.

15. HUGHES (T. E.) (1959). - Mites, or the Acari. London, $225 \mathrm{p}$.

16. KATES (K. C.) et RUNKEL (C. E.) (1948). Observations on Oribatide mite vectors of Moniezia expansa on pasture with a report of several new vectors from the. U. S. Proc. Helm. Soc. Wahs., 15, 1, 19-33.

17. KHOLOSHCHANOV (V. A.) (1955). - Epizootiology of Monieziasis and mesures for controlling it. Vet., Moscou, 32, 4, 33-5.

18. KRULL (W. H.) (1939 a). - Observations on the distribution and ecology of the Oribatid mites. J. Wosh. Acod. Sci., 29, 12, 519-28.

19. KRULL (W. H.) (1939 b). - On the lifeHistory of Moniezia and Cittotaenia. Proc. Heim. Soc. Wash., 6, 1, 10-11.

20. KUZNETZOV (M. I.) (1962), - The intermediate hosts of Thysaniezia and Avitellina in sheep. Vet., Moscou, 39, 7, 46-7.

21. MEHRA (K. N.) et SRIVASTATA ( $H$. D.) $(1955, a)$. - Studies on the life history of Moniezia benedeni, a tapeworm of ruminants. Proc. Ind. Scl. Congr., III, 352.

22. MEHRA (K. N.) et SRIVASTATA ( $H$. D.) (1955 b). - Studies on the life history of Moniezia expansa, a broad tapeworm of ruminants. Proc. Ind. SCl. Congr., III, 352.

23. MOREL (P.) (1953). - Les Cestodes du mouton. Thèse vétérinaıre, Parıs.

24. NADAKAL (A. M.) $(1960,0)$. - Observation on the life-cycle of Avitellina centripunctata (Riv. 1874) an Anoplocephalinae Cestode from sheep and goats. J. Porosit,. 46, 5 (Sect. 2), 12.

25. NADAKAL (A.M.) $(1960$, b). - Protoscheloribates sp., an Oribatid mite from India as a potential vector of the sheep Tapeworm Moniesia benedeni and $M$. expansa. J. Parasit., 46, 6, 817.

26. NADAKAL (A. M.) (1961). - Structure and development of paruterine orgarns in Avitellina centripunctata. J. Parasit., 47, 4(sect. 2), 57.

27. OREKHOV (M. D.) (1960). - Epizootiology of Moniezia in sheep and goast and its control in the Turkmen S. S. R. Trud. Turkmen. 
Nauchno-issledovat. Inst. Zhivotnov I. Vet., 2, 267-88.

28. PETROCHENKO (V. I.) (1954). - The role played by mites and insects in the epizootiology of Helminth diseases. Probl. Vet. Dermat. Arakhnol. Entomol., 1, 22-3.

29. POTEMKINA (V. A.) (1941), - Contribution to the biology of Moniezia expansa. C. R. AC. Sci. U. R. S. S., IXXX, 5, 474-6.

30. POTEMKINA (V. A.) (1944), 一 On the decipherment of the biological cycle in Moniezia benedeni. C. R. Ac. Sci. U.R. S. S., XLII, 3, $146-8$.

31. POTEMKINA (V. A.) $(1944$, b). - Contribution to the study of the development of Thysaniezia ovilla. C. R. Ac. Sci. U. R. S. S. XLII!, I, 43-4.

32. POTEMKINA (V. A.) (1959). - The Epizootiology of Monieziasis in ruminants. Trud. Vsesoyoz. Inst. Gelmint. Im K, I. Skrjabin, 6, 50-6.

33. RAJSKI (A.) (1959). - Moss-mites (AcariOribatei) as intermediate hosts of Anoplocephalata. Rev. Zesz. Nauk. Uniwer. Im. A. Micklewicza II : 163-92.

34. RAO (N. S. K.) ef CHOQUETTE (L. P. E.) (1951), - An intermediate host of Moniezia expansa in Earstern Quebec. Canad. J. Comp. Med. Vet. Sci., 15, 12-14.

35. RAYSKI (C.) (1949). - Observations on the life history of Moniezia, with special reference to the bionomics of the Oribatid mites. XIV th Int. Vet. Cong. London, 11, 51-55.

36. ROBERTS, (F. H. S.) (1953). - Zygoribatula longiporosa, an intermediate host of Moniezia benedeni in Australia. Austr. J. Zool., $1 ; 2$; $239-41$.

37. RUKAVINA (J.) et ALL (1960). - Stanje zdravlja ovaca na nekim poljoprivrednim dobrima. Rezultati komplesksnich istrazivanja stanja paraziparnih i zar-aznih oboljenja, te analiza krvi ovoca i stocne hrane u 1959 godinì na nekim poljoprivrednim dobrima u Bosni. Vet., Sorajevo, 9, 3, 497-514.

38. SOKOLOVA (1. B.) et PANIN (V. Y.) (1960). - The intermediate hosts of Moniezia Thysaniezia et Avitellina in Kazakstan (en russe). Trud. Inst. Zool. Akad. N. Kazakh. S. S. R., 12, 145-9.

39. STUNKARD (H. W.) (1937). - The life cycle of Moniezia expansa. Science, $L X X X V I, 312$.

40. STUNKARD (H. W.) (1938). - The development of Moniezia expansa in the intermediate host. Parasit., 30, 4, 491-501.

41. STOLL (N. R.) (1935 a). - Tapeworm studies I. Restricted pastures as sources of Moniezia infection in sheep. Am. J. hyg., 21, 628-46.

42. STOLL (N. R.) (1935 b). - Tapeworm studies II. Persistance of the posture stoge of Moniezio expansa. Hyg., 22, 3, 683-703.

43. SVADZHYAN (P. K.) (1962), - Species composition of Oribatid mites, intermediate hosts of Moniezia, their distribution and natural infection in Armenian S. S. R. Zool. Sborn. AK. N. Armyansk S. S. R., 12, 163-178.

44. WALLWORK (J. A.) et RODRIGUEZ (J. G.) (1961). - Ecological studies on Oribatid mites, with particular reference to their role as intermediate hosts of Anoplocephalid Cestodes. J. Econ. Ent., 54, 4, 701-5.

45. WALLWORK (J. A.) 1964), -- Rev. Zool. Bot. Afr. (en préparation).

46. WATANABE et COLL (1957). - - Studies on the intermediate host of Moniezia expansa. 1. J.Vet. Med. Ass., 10, 12, 582-5.

47. WHARTON (G. W.) (1952), - An introduction to Acarology. New York, 465 pp, 377 figs

\section{ADDENDUM}

Depuis la remise de ce texte pour publication, nous avons eu connaissance d'un travail de R.S. Tandon paru dans la revue Parasitologia de décembre 1963 (V, 3, 183-187).

L'auteur, à Lucknow aux Indes, décrit le cycle évolutif de Stilesio globipunctata dont l'hôte intermédiaire est Scheloribates indica. Les essais d'infestation ont été effectués sur des Oribates élevés au laboratoire. 